\title{
Calculation of degassing networks taking into account the accumulation of the liquid phase
}

\author{
Leonid Novikov ${ }^{1, *}$, and Oleksandr Bokii ${ }^{2}$ \\ ${ }^{1}$ Institute of Geotechnical Mechanics named by N. Poljakov of National Academy of Sciences of \\ Ukraine, 49005, Dnipro, Simferopolska Str., 2a, Ukraine \\ ${ }^{2}$ Limited Liability Company "DzhiI Ukraine", 01004, Kyiv, Shovkovichna Str., 42/44, Ukraine
}

\begin{abstract}
The issue of accounting for the accumulation of liquid in the mine degassing network during gas-dynamic calculations is considered. Geometry of fluid accumulation in the cross section of degassing pipeline and the formulas for geometric parameters are presented. A scheme of wave generation on the liquid surface is considered. Dependence for the coefficient of resistance to interfacial friction on the liquid surface is proposed. Formulas for calculation of gas-dynamic parameters on the pipeline section are given. The results calculation of the resistance coefficients in the place accumulation of liquid, changes in flow and pressure of the gas mixture are presented. Calculations carried out for pipelines with accumulations of liquid.
\end{abstract}

\section{Introduction}

The operation of the mine degassing of system is characterized by the interaction of its main elements with each other. The efficiency of degassing depends on the nature of the gas-dynamic processes in the degassing network. The unsatisfactory condition of mine degassing pipelines is caused by a decrease in their tightness, damage to the walls, as well as their contamination by distributed and local sediments. This leads to an increase in the total aerodynamic resistance of degassing network [1-3], and growth in the cost of electricity for transporting the gas mixture and an increase in the concentration of methane in mining. When designing degassing of systems, local resistances in the degassing network are taken into account by increasing the length for pipes by $10 \%$ or more. At the same time influence of liquid phase accumulations formed as a result of condensation processes and liquid from degassing wells is not fully taken into account. These accumulations lead to noticeable fluctuations in vacuum, absolute pressure and flow of the gas mixture in the pipelines. In the case of complete closure of the pipeline section, these fluctuations reach a maximum. This disrupts the process of transporting the gas mixture and increases the likelihood of accidents. In this regard, when carrying out gas dynamic calculations at the design and reconstruction stage of the degassing system, it is necessary to take into account the factor of moisture accumulation [1].

\footnotetext{
*Corresponding author: lnov710@gmail.com
} 


\section{Methods}

When calculating the degassing pipeline, the following gas dynamic parameters are determined: density and flow rate of gas mixture; speed of gas mixture and concentration of methane; absolute pressure and vacuum in the pipeline; aerodynamic resistance for pipeline; temperature of the gas mixture.

Initial data for the calculation of degassing system: gas flow; type of local resistance; degassing wells; fluid intensity from wells; length and diameter of the pipeline (Fig. 1).

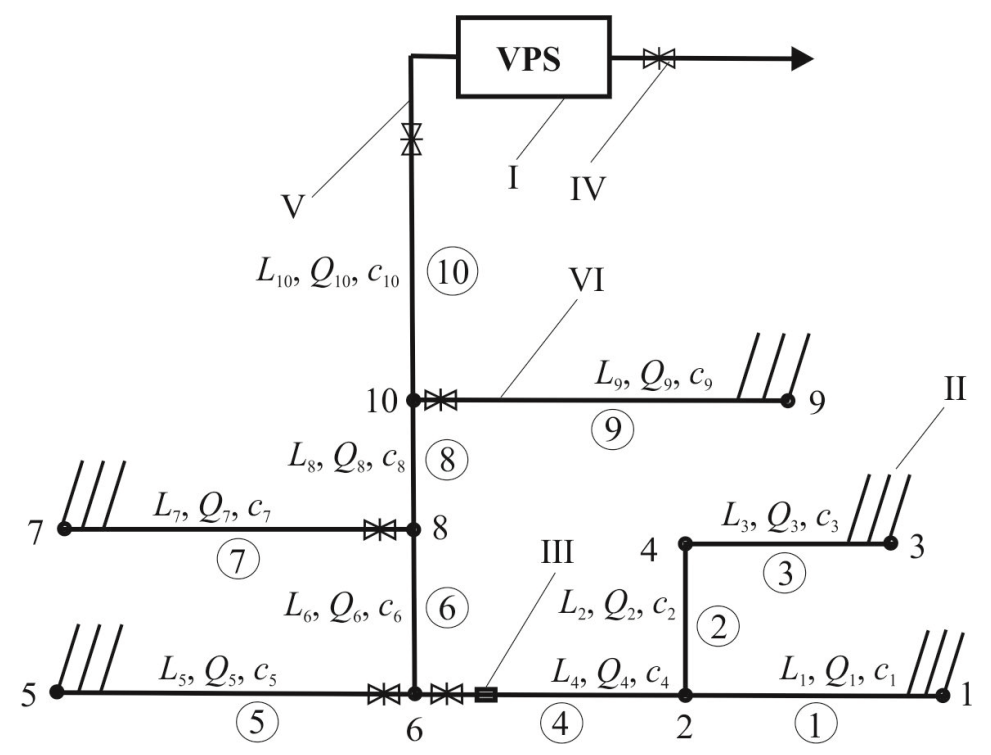

Fig. 1. Degassing network: 1, 2 - 10 - nodes; 1,2 - 10 - branches; $L_{i}$ - lengths of branches, m; $c_{i}-$ methane concentration, $\%, Q_{i}-$ gas mixture flow rate, $\mathrm{m}^{3} / \mathrm{s}$; I - vacuum pumping station (VPS); II degassing wells; III - device for draining water; IV - adjusting valve; V - main pipeline; VI precinct pipeline.

During the calculations, the parameters of the gas mixture in the branches and nodes of the network are determined; efficiency of the vacuum pumps is checked. According to the results, adjustment of the design scheme is carried out.

Presence of the water in degassing network, as well as the flow of air through the flange connections of the pipeline lead to a change in values of resistance coefficients and density of gas mixture. In turn, this leads to corresponding changes in other gas-dynamic parameters.

Figure 2 illustrates the form of fluid accumulation in the pipeline cross section.

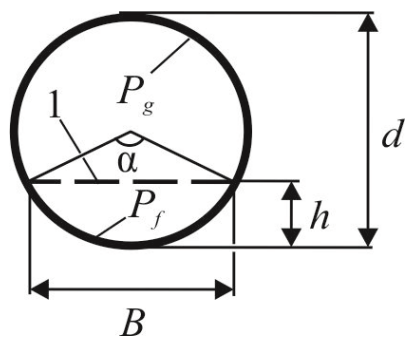

Fig. 2. Form of liquid accumulation in cross section pipeline: 1 - free surface of liquid; $P_{g}, P_{f}-$ pipeline perimeters, respectively, for gas and liquid, $\mathrm{m} ; h$ - liquid level, $\mathrm{m} ; \alpha-$ central angle, rad; $B-$ fluid accumulation width, $\mathrm{m} ; d$ - pipeline internal diameter, $\mathrm{m}$. 
A segment of liquid accumulation is presented in Figure 2. For a segment, you can write the following relations:

$$
\begin{gathered}
P=P_{g}+B ; \\
B=2\left(h d-h^{2}\right)^{0.5} ; \\
P_{g}=0.5 d(2 \pi-\alpha)=0.5 d\left[2 \pi-2 \arccos \left(1-2 d^{-1} h\right)\right] \\
S_{g}=0.25 \pi d^{2}-S_{f} ; \\
d_{g}=4 S_{g} P_{g}^{-1} ; \\
d_{z}=4 S_{g} P^{-1} ; \\
\operatorname{Re}_{g}=\frac{Q d_{g} \rho}{S_{g} \mu} ; \\
\operatorname{Re}_{z}=\frac{Q d_{z} \rho}{S_{g} \mu}
\end{gathered}
$$

where $P$ is perimeter of the passage cross section of the pipeline, $\mathrm{m} ; S_{g}$ is area of passage section of the pipeline, $\mathrm{m}^{2} ; S_{f}$ is cross sectional area of liquid accumulation, $\mathrm{m}^{2} ; d_{g}$ is hydraulic diameter of the pipeline, $\mathrm{m} ; d_{z}$ is hydraulic diameter for the passage section, $\mathrm{m}$; $R e_{g}$ is Reynolds number for the gas perimeter; $R e_{z}$ is Reynolds number for perimeter of the passage section of the pipeline; $\rho$ is density of the gas mixture, $\mathrm{kg} / \mathrm{m}^{3} ; \mu$ is dynamic viscosity of the gas mixture, $\mathrm{kg} /(\mathrm{m} \cdot \mathrm{s}) ; Q$ is flow rate of the gas mixture; $\mathrm{m}^{3} / \mathrm{s}$.

A value of the total coefficient of friction resistance at the place of accumulation of liquid [4] is determined by the formula [1,2]:

$$
\xi_{s u m}=\xi_{t}+\xi_{f}
$$

where $\xi_{t}$ is coefficient of friction resistance; $\xi_{f}$ is coefficient of resistance to interfacial friction [4].

A coefficient of friction resistance for a clean pipeline is determined by the formula $[2,5]$ :

$$
\xi=\frac{\lambda L_{t}}{d}=0.11\left(\frac{\Delta}{d}+\frac{68}{R e}\right)^{\frac{1}{4}} \frac{L_{t}}{d},
$$

were $\lambda$ is Darcy coefficient; $\Delta$ is absolute equivalent roughness for internal surface of the pipeline, $\mathrm{m}$; $R e$ is Reynolds number $\left(10 \Delta^{-1} d<R e<500 \Delta^{-1} d\right) ; L_{t}$ is pipeline length, $\mathrm{m}$.

For fluid accumulation, the expression (10) takes the view:

$$
\xi_{t}=0.11\left(\frac{\Delta}{d_{g}}+\frac{68}{R e_{g}}\right)^{\frac{1}{4}} \frac{L_{s}}{d_{g}},
$$

were $L_{s}$ is length of accumulation, $\mathrm{m}$.

Suppose that the wave surface of liquid (small waves) is similar to the roughness for inner surface of degassing pipeline.

In Figure 3 the shape of wave surface is presented. 


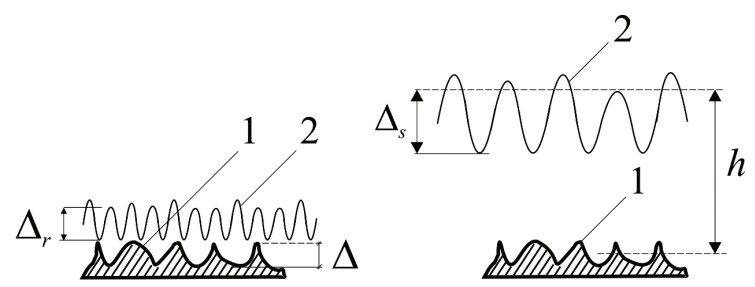

Fig. 3. Formation of waves on the free surface of the liquid in the pipeline: 1 - protrusions roughness; 2 - wave surface; $\Delta_{r}, \Delta_{\mathrm{s}}$ - wave heights on free surface of film water and fluid accumulation, $\mathrm{m} ; h-$ liquid level, $\mathrm{m}$.

The maximum roughness of the inner surface of the pipeline $\Delta=\Delta_{\max } \approx 2-2.5 \mathrm{~mm}$. This is typical for degassing pipes after long-term operation (effect of corrosion processes).

The total coefficient of friction resistance can be determined by the formula:

$$
\xi_{\text {sum }} \approx 0.11\left(\frac{\Delta}{d_{z}}+\frac{68}{R e_{z}}\right)^{0.25} \frac{L_{s}}{d_{z}}
$$

Using the expression (12) it is possible to obtain an approximate dependence of the coefficient of resistance to interfacial friction:

$$
\xi_{f}=\frac{\lambda_{f} L_{s}}{4 S_{g} B^{-1}} \approx \xi_{\text {sum }}-\xi_{t}=0.11 L_{s}\left[\left(\frac{\Delta}{d_{z}}+\frac{68}{R e_{z}}\right)^{0.25} \frac{1}{d_{z}}-\left(\frac{\Delta}{d_{g}}+\frac{68}{R e_{g}}\right)^{0.25} \frac{1}{d_{g}}\right],
$$

where $\lambda_{f}$ is interfacial friction loss coefficient (analogue $\lambda$ ).

Based on the analysis of accumulation form $[1,5]$, the formula for cross-sectional area of the degassing pipeline is obtained:

$$
S_{g}=\frac{d^{2}}{8}\left\{2 \pi-2 \arccos \left(1-\frac{2 h}{d}\right)-\sin \left[2 \pi-2 \arccos \left(1-\frac{2 h}{d}\right)\right]\right\},
$$

where $h=a V^{b} ; V$ is volume of liquids, $\mathrm{m}^{3} ; a, b$ are numeric coefficients.

The expression (14) allows connecting the cross-sectional area of the pipeline with the liquid level and its volume. A volume and level of liquid are determined experimentally.

The degassing pipeline consists of links. It should be borne in mind that the air enters the pipeline through the flange connections of the links.

For an arbitrary link of the pipeline, we write down the relations for the following gasdynamic parameters:

$$
\begin{gathered}
p_{k}=\sqrt{p_{n}{ }^{2}-p_{n} \frac{\rho_{n} Q_{n}{ }^{2}}{S_{g}^{2}} \xi_{\text {sum }}} ; \\
Q_{k}=\left(Q_{n}+\Delta Q\right) \frac{p_{k} T_{0}}{p_{a} T_{k}} ; \\
\rho_{k}=\rho_{v}\left(1-c_{k}\right)+\rho_{m} c_{k},
\end{gathered}
$$

where $p_{n}$ is absolute pressure of the gas mixture in the initial cross section of the pipeline link, $\mathrm{kg} /\left(\mathrm{m} \cdot \mathrm{s}^{2}\right) ; p_{k}$ is absolute pressure of the gas mixture in the final cross section 
of the pipeline link, $\mathrm{kg} /\left(\mathrm{m} \cdot \mathrm{s}^{2}\right) ; Q_{n}$ is gas mixture flow rate in the initial cross sectional of the pipeline link, $\mathrm{m}^{3} / \mathrm{s} ; Q_{k}$ is gas mixture flow rate in the final cross sectional of the pipeline link, $\mathrm{m}^{3} / \mathrm{s} ; p_{a}$ is air pressure under normal conditions, $\mathrm{kg} /\left(\mathrm{m} \cdot \mathrm{s}^{2}\right) ; T_{0}$ is air temperature under normal conditions, $\mathrm{K} ; T_{k}$ is temperature of the gas mixture in the final cross section of the pipeline link, $\mathrm{K} ; \Delta Q$ is inflow of air for the link of pipeline, $\mathrm{m}^{3} / \mathrm{s} ; \rho_{n}$ is density of the gas mixture in the initial cross section of the pipeline link, $\mathrm{kg} / \mathrm{m}^{3} ; \rho_{k}$ is density of the gas mixture in the final cross section of the pipeline link, $\mathrm{kg} / \mathrm{m}^{3} ; \rho_{v}, \rho_{m}$ are air and methane density, $\mathrm{kg} / \mathrm{m}^{3} ; c_{k}$ is concentration of methane at the end of the pipeline link, \%.

When calculating degassing pipelines, it is necessary to take into account the presence of fluid accumulations and changes in the parameters of the gas mixture due to air inflows. To improve the accuracy, a sequential calculation of each pipeline link is carried out.

In Figure 4 presents the classification of methods for calculating networks of pipelines.

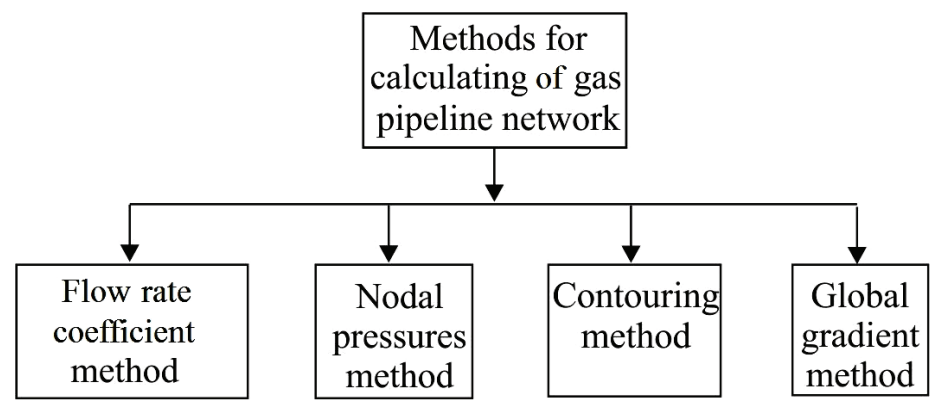

Fig. 4. Classification of calculation methods gas pipelines.

Flow rate coefficient method takes into account the change in the capacity of the gas pipeline in comparison with the reference pipeline. Nodal pressure method allows determining the gas pressure in the network nodes. Contouring method is used to consider a network graph indicating the direction of gas flow. Global gradient method allows calculating networks with different configurations. In this case, the gas flow and pressure values are set at the network nodes. The considered methods use assumptions about stationary and isothermal gas flow. The disadvantages of the methods are the occurrence of errors in the calculation of gas-dynamic parameters for local resistances (including fluid accumulations), as well as for the connections of pipelines of different diameters.

\section{Results and discussion}

During the calculations, a degassing pipeline with an internal diameter of 0.257 and $0.309 \mathrm{~m}$ was considered. The velocity of the gas mixture is $U=10-15 \mathrm{~m} / \mathrm{s}$. The liquid level in the place of its accumulation is $h=0.5 d$.

Table 1 presents the results calculation of Darcy coefficient and the coefficient of loss for interfacial friction.

Table 1. Resistance coefficients for degassing pipeline.

\begin{tabular}{|c|c|c|c|c|c|}
\hline$d, \mathrm{~m}$ & $U, \mathrm{~m} / \mathrm{s}$ & $\Delta, \mathrm{m}$ & $R e \cdot 10^{5}$ & $\lambda$ & $\lambda_{f}$ \\
\hline \multirow{4}{*}{0.257} & & 0.00005 & $1.36-2.0$ & $0.018-0.017$ & - \\
\cline { 3 - 6 } & & 0.001 & $1.35-1.99$ & $0.0283-0.028$ & - \\
\cline { 3 - 6 } & \multirow{4}{*}{0.15} & 0.002 & $1.35-1.98$ & 0.033 & 0.056 \\
\cline { 3 - 6 } & & 0.00005 & $1.63-2.41$ & $0.02-0.015$ & - \\
\cline { 3 - 6 } & & 0.001 & $1.63-2.40$ & 0.027 & - \\
\cline { 3 - 6 } & & 0.002 & $1.63-2.39$ & $0.032-0.031$ & $0.053-0.054$ \\
\hline
\end{tabular}


From the analysis of Table 1 it follows that the value of the coefficient of loss in interfacial friction exceeds the value of Darcy coefficient by 1.7 times.

Figure 5 presents the results of the calculation of the volume flow of the gas mixture on area of degassing pipeline with the accumulation of liquid.

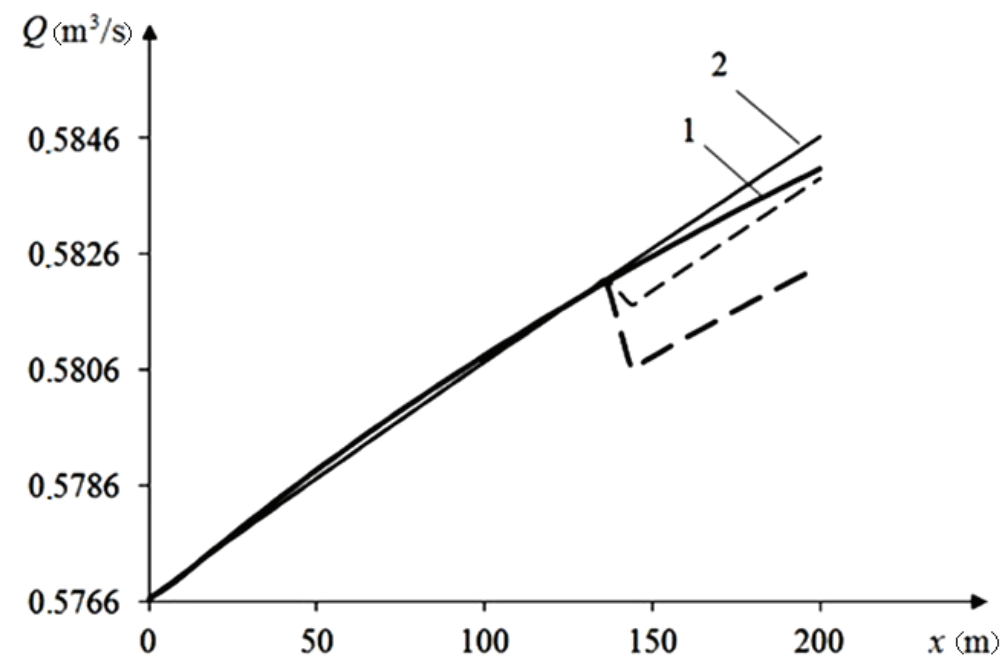

Fig. 5. Changes in the flow rate of the gas mixture along the length of the degassing pipeline section with fluid accumulation: ---- - flow rate of behind an accumulation; $1-d=0.257 \mathrm{~m} ; 2-d=0.309 \mathrm{~m}$.

Analysis of figure 5 shows that the decrease in the flow rate of gas mixture in the place of liquid accumulation is due to pressure losses. At the same time influence of the gas flow leads to the generation of wave disturbances in the liquid. This results in periodic overlap of pipeline.

Figure 6 shows the flow characteristics of the pipeline at different values of absolute equivalent roughness of its inner surface.

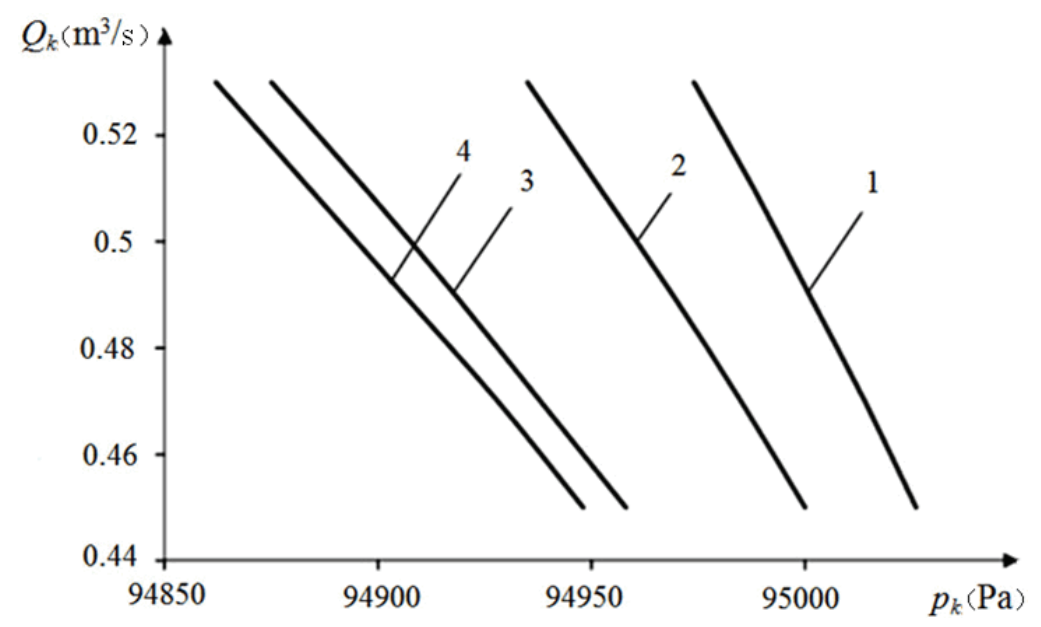

Fig. 6. Flow characteristics for pipeline at various values of roughness of its inner surface: $1-\Delta=$ $0.00005 \mathrm{~m} ; 2-\Delta=0.0003 \mathrm{~m} ; 3-\Delta=0.001 \mathrm{~m} ; 4-\Delta=0.001 \mathrm{~m}$ and fluid accumulation.

Analysis of Figure 6 shows that the flow rate of gas mixture is inversely proportional to the absolute pressure at the end of the pipeline. With an increase internal roughness of the pipeline, its flow characteristic is shifted to the area of lower pressures. 


\section{Conclusions}

On the basis of research, the following conclusions can be made:

1. When determining the coefficients of friction resistance in degassing pipelines it is necessary to take into account the interaction of the gas mixture flow with the free surface of the liquid. In this case low-intensity wave perturbations of liquid are approximately considered as analogues of the roughness for solid surface.

2. At the given values of the conditional roughness for free surface of liquid in the degassing pipeline the value of the loss coefficient for interfacial friction exceeds the value of Darcy coefficient on average by 1.7 times.

3. A sharp drop in the flow rate of gas mixture is associated with the generation of wave perturbations liquid which periodically overlap the cross section of degassing pipeline.

\section{References}

1. Novikov, L.A. (2014). Impact of disperse phase on hydraulic resistance of district degassing pipelines, Geotechnical Mechanics, (114), 154-161

2. Krivoshchekov, V.I., Novikov, L.A. (2016). Zakonomernosti izmeneniya poter davleniya $v$ truboprovode nizkogo davleniya, Zbahachennya korysnykh kopalyn, 64, (105), 26-29

3. Mukha, O.A., Puhach, I.I. (2009). Rozrahunok parametriv degazatsiynih system. Dnipropetrovsk: NMU

4. Petukhov, B.S., Henin, L.G., Kovalev, S.A., Solovyev, S.L. (2003). Teploobmen v yadernyih energeticheskih ustanovkah. Moskva: MEI

5. Novikov, L.A. (2015). Gas dynamics flooded areas degassing pipeline and methods of calculation of parameters, Geotechnical Mechanics, (120), 234-243

6. Shkundin, S.Z., Ivannikov, A.L., Zinchenko, I.N. (2011). Coal mines degassing nets modeling by inter ventilation net nodes method, Gornyy informatsionno-analiticheskiy byulleten, (9), 397-401 